\title{
Microstructural Characteristics and Mechanical Properties of Water Cooling Bobbin-Tool Friction Stir Welded 6063-T6 Aluminum Alloy
}

\author{
Yunqiang Zhao, Chungui Wang and Chunlin Dong \\ Guangdong Provincial Key Laboratory of Advanced Welding Technology, Guangdong Welding Institute (China-Ukraine E.O.Paton Institute \\ of Welding), Guangzhou, 510650, China
}

\begin{abstract}
In this study, a novel welding method called water cooling bobbin-tool friction stir welding (WBT-FSW) was developed. 4 mm-thick 6063-T6 aluminum alloy sheets were successfully jointed by WBT-FSW. Comparative studies on macro/microstructural characteristics and mechanical properties of the WBT-FSW and conventional bobbin-tool friction stir welding (BT-FSW) joints were carried out. The results indicated that the water mist cooling can significantly decrease the welding temperature and improve both the weld formation and the mechanical properties of the joint. The tensile strength of the WBT-FSW joint was $11.4 \%$ higher than that of BT-FSW joint.
\end{abstract}

\section{Introduction}

Friction stir welding (FSW) is a solid state joining technology, and it is quite suitable for joining aluminum alloys [1-3]. However, the curved and hollow sections cannot be joined by FSW, because a back plate is necessary to support the workpiece during welding [4]. In order to overcome this problem, bobbin-tool friction stir welding (BT-FSW) has been developed. For this bobbin tool, top and bottom shoulders jointed by the pin are applied, so the workpiece can be supported by this lower shoulder instead of the back plate.

Until now, some studies on the BT-FSW of aluminum alloys have been carried out in order to obtain high quality joint. For instance, Liu et al. [5] studied the BTFSW of 6061-T6 aluminum alloy and they found that the precipitates deterioration caused by the welding heat input was the main softening mechanism of the joint. In addition, Al-Mg-Si alloy sheets were bobbin-tool friction stir welded using a specially designed tool by Zhou et al. [6]. The authors found that the lowest hardness location was in the heat affected zone of the joint and both the lowest hardness value and the tensile strength of the joint were increased with decreasing the welding heat input. Zhang et al. [7] conducted BT-FSW experiments on 2A14-T6 aluminum alloy sheets. The result also indicated that the strength of the joint can be increased by increasing the welding speed. It can be conclude that decreasing the welding heat input is an effective means for improving the mechanical properties of the BT-FSW joint. However, the effect by optimizing welding parameters is limited, because weld defect will be formed if the material cannot be sufficiently mixed at too high welding speed or too low tool rotation speed $[8,9]$. In order to effectively decrease the welding heat input without changing the welding parameters, external water cooling was applied in the conventional FSW process in some studies [10]. The results indicated that the external water can take away the welding heat and then increase the mechanical properties of the FSW joints. For instance, Fratini et al. [11] applied water cooling on the top surface of the workpiece in the FSW experiment of 7075-T6 aluminum alloy, It was found that the tensile strength of the joint can be improved by the water cooling effect. Zhang et al [12-15] conducted the conventional FSW experiments on 2219-T6 aluminium alloy, during which the whole workpiece was immersed in the water environment. The results indicated that water cooling can be utilized to improve the mechanical properties of the FSW joint.

For increasing the joint strength, water cooling has been applied in the conventional FSW process. However, for the BT-FSW which suffers from high welding heat input and heat softening effect, the effects of the water cooling on the welding heat input and mechanical properties of the joint has not been studied. In this study, in order to effectively decrease the welding heat input and increase the strength of the BT-FSW joint, water spraying was applied during BT-FSW. This novel BTFSW method was defined as water cooling bobbin-tool friction stir welding (WBT-FSW). 6063-T6 aluminum alloy which is widely applied in high-speed train structures was joined by both BT-FSW and WBT-FSW with the aim of highlighting the advantages of this novel welding method. The focus is placed on the weld formation, macro/microstructural characteristics, hardness distributions and tensile properties. 
Table 1 Chemical compositions mechanical properties of 6063-T6 aluminum alloy

\begin{tabular}{ccccccccccccc}
\hline \multicolumn{1}{c}{ Chemical compositions, wt. \% } & \multicolumn{4}{c}{ Mechanical properties } \\
\hline $\mathrm{Mg}$ & $\mathrm{Si}$ & $\mathrm{Fe}$ & $\mathrm{Mn}$ & $\mathrm{Cr}$ & $\mathrm{Zn}$ & $\mathrm{Cu}$ & $\mathrm{Ti}$ & $\mathrm{Al}$ & Tensile strength & Elongation \\
$0.45 \sim 0.9$ & $0.2 \sim 0.6$ & 0.35 & 0.1 & 0.1 & 0.1 & 0.1 & 0.1 & $\mathrm{Bal}$ & $260 \mathrm{Mpa}$ & $12 \%$ \\
\hline
\end{tabular}

After welding, the metallographic specimens were

\section{Material and Experiment}

4 mm-thick 6063-T6 aluminum alloy was taken as the base material whose chemical compositions and mechanical properties are listed in Table 1. For the bobbin tool, the diameters of both top and bottom shoulders were $16 \mathrm{~mm}$, and the shoulder surfaces were flat with spiral groove. A cylinder pin with two symmetric threads was applied and its diameter and length were $8 \mathrm{~mm}$ and $4 \mathrm{~mm}$ respectively. During both BT-FSW and WBT-FSW, the tool rotation speed and welding speed were fixed at $1200 \mathrm{rpm}$ and $200 \mathrm{~mm} / \mathrm{min}$ respectively. The plunge depth of both top and bottom shoulders was set to $0.05 \mathrm{~mm}$. In the WBT-FSW process, water mist with a constant flow rate of $0.1 \mathrm{~L} / \mathrm{min}$ was sprayed to the leading edge of the top shoulder, as shown in Figure 1a. In order to measure the welding thermal cycles, two K-type thermocouples were embedded into the workpiece on the advancing side (AS) of the weld with a depth of $2 \mathrm{~mm}$. Their distances from the weld center were $9 \mathrm{~mm}$ and $12 \mathrm{~mm}$ respectively, as illustrated in Figure 1b.
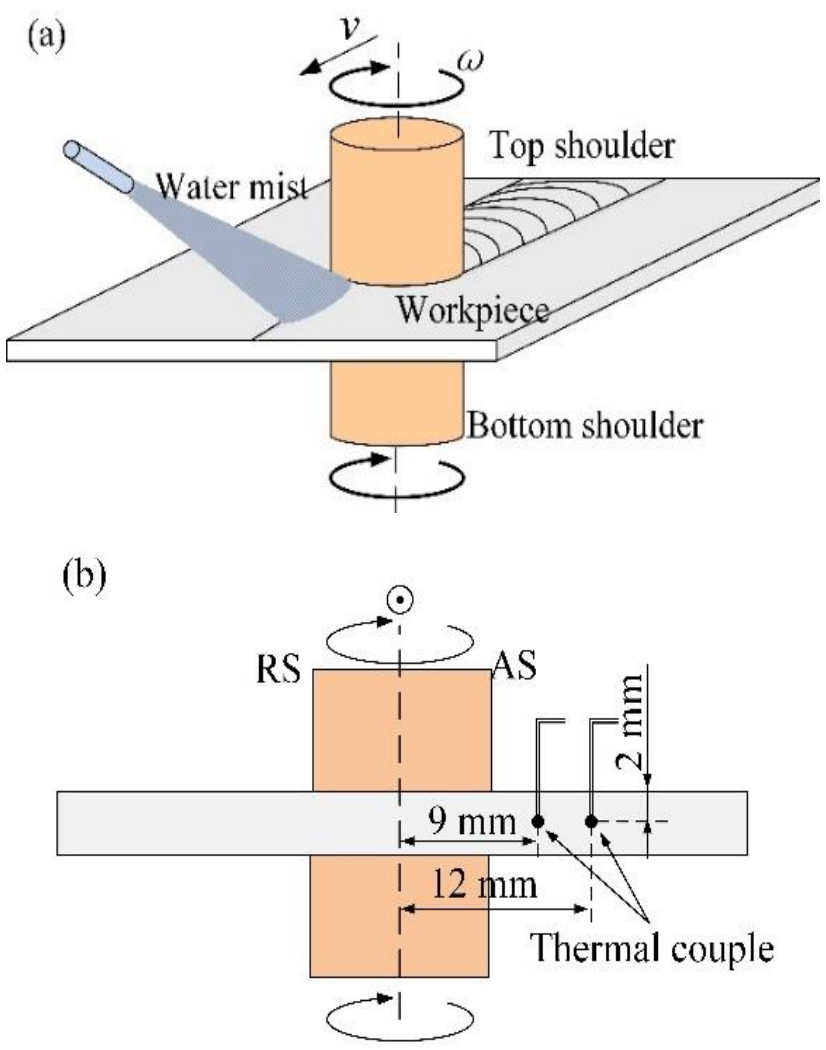

Figure 1. Schematic illustrations of (a) WBT-FSW process and (b) thermal couple positions successively sanded, polished and then etched using Keller's reagent. The metallographic analyses were carried out by optical microscopy (OM, Olympus-MPG3). Microhardness distributions on the cross-sections of the joints were measured along three lines whose distances from the workpiece top surface were $1 \mathrm{~mm}, 2 \mathrm{~mm}$ and $3 \mathrm{~mm}$ respectively. The space between the adjacent indentations was $0.5 \mathrm{~mm}$, and the testing load was $1.96 \mathrm{~N}$ for $10 \mathrm{~s}$. The tensile tests were carried out using an electron universal testing machine (Instron 1186). The fracture morphologies of the failure specimens were observed using scanning electron microscope (SEM, Hitachi-S3400N).

\section{Results and Discussions}

\subsection{Thermal cycles.}

For the heat-treatable aluminum alloys, the welding thermal cycle can lead to precipitates deterioration and grain coarsening [16]. This is the main weakening mechanism for the BT-FSW or FSW joints [17]. For the 6063 aluminum alloy in this study, the main strengthening phase is $\beta^{\prime \prime}$ which are coherent with the aluminum matrix. In addition, the $\beta^{\prime}$ phase which is semicoherent with the matrix has weaker strengthening effect

[18]. The artificial aging temperature of 6063 aluminum alloy is $175^{\circ} \mathrm{C}$ at which the $\beta^{\prime \prime}$ particles are accelerated to precipitate. When the temperature is in the range of $207-$ $250{ }^{\circ} \mathrm{C}$, the small $\beta$ " dissolution and the large $\beta$ " growth will occur. When the temperature is in the range of 250 $320{ }^{\circ} \mathrm{C}$, the $\beta^{\prime \prime}$ precipitates will be coarsened and transform into $\beta^{\prime}$ precipitates. With the temperature further increasing in the range of $400-480{ }^{\circ} \mathrm{C}$, the $\beta^{\prime}$ precipitates will be dissolved into the matrix.

Figure 2 shows the welding thermal cycles at different positions during BT-FSW and WBT-FSW. The measured positions are respectively located in the thermomechanically affected zone (TMAZ) and the heataffected zone (HAZ) of the weld. For the BT-FSW, the peak temperature and the dwelling time at high temperature (artificial aging temperature, $175{ }^{\circ} \mathrm{C}$ ) in the TMAZ are $419{ }^{\circ} \mathrm{C}$ and $10 \mathrm{~s}$ respectively. It can be concluded that the $\beta^{\prime \prime}$ precipitates in the TMAZ experience dissolution and transformation into the $\beta$ precipitates. After that, the $\beta^{\prime}$ should be coarsened and partially dissolved into the matrix with the temperature increasing. In the HAZ of the BT-FSW joint, the peak temperatures and the dwelling time at high temperature are $309{ }^{\circ} \mathrm{C}$ and $8.7 \mathrm{~s}$ respectively. The $\beta$ " precipitates in this zone should be partially dissolve and the large $\beta "$ precipitates should transform into the $\beta^{\prime}$ precipitates. In 
the WBT-FSW process, the measured peak temperatures and the dwelling time at high temperature were dramatically decreased. This can be attributed to the significant cooling effect of the water mist. In addition, the measured welding thermal cycles in the WBT-FSW process are more fluctuant, owing to the instability of the water mist cooling. For the WBT-FSW, the peak temperature and dwelling time at high temperature in the TMAZ are $278{ }^{\circ} \mathrm{C}$ and $2.8 \mathrm{~s}$ respectively. It can be concluded that the $\beta^{\prime \prime}$ precipitates should partially dissolve and transform into the $\beta^{\prime}$ precipitates. The peak temperature and dwelling time at high temperature in the HAZ are $197{ }^{\circ} \mathrm{C}$ and 0.5 s respectively. As a result, only $\beta "$ precipitates coarsening should occur in this zone.

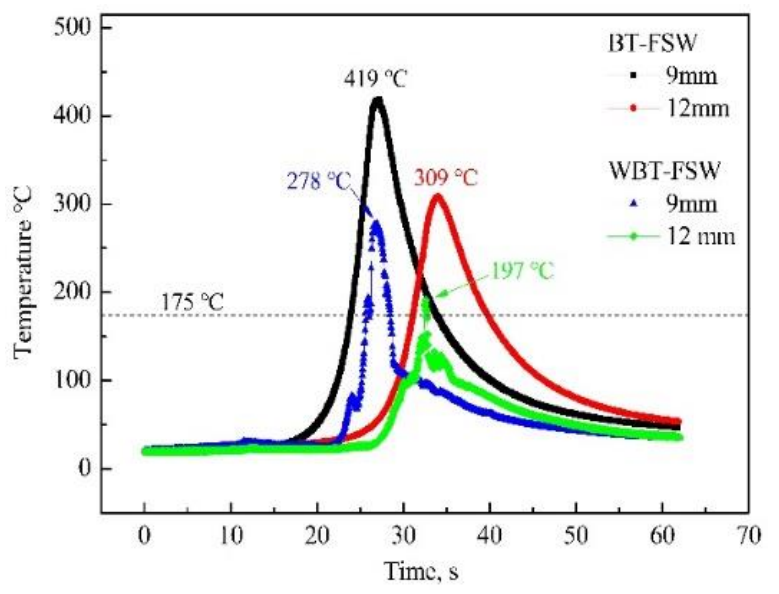

Figure 2. Welding thermal cycles with different distances from weld center in BT-FSW and WBT-FSW

\subsection{Macro/microstructural characteristics.}

As shown in Figure 3, defect-free joints can be obtained by both BT-FSW and WBT-FSW. For BT-FSW, the weld surface is rough and superfluous flash is formed at the retreating side (RS) of the weld (Figure 3a). This can be attributed to the softened material with low viscosity extruded out from the top and bottom shoulder at high temperature. By contrast, the weld surface obtained by WBT-FSW is smoother and the superfluous flash is vanished (Figure 3b), owing to the cooling effect of the water mist.

Figure $3 \mathrm{c}$ and d display the cross-section macrographs of BT-FSW and WBT-FSW joints. For both BT-FSW and WBT-FSW, the geometry of the SZ displays a dumbbell-shape. The width of the SZ at the midthickness is a little larger than the pin diameter, and the widths of the top and the bottom surfaces are almost equal to the shoulder diameter. The transition between the SZ and the TMAZ on the advancing side (AS) is sharper than that on the RS. Mishra et al. [19] ascribed this to the different flow behaviours and the degree of plastic deformation of the material on both sides of welding tool. For the WBT-FSW joint, the water mist cooling suppresses the flow of the plastic material, as a result, the sizes of the SZ, the TMAZ and the HAZ are obviously narrower than those in the BT-FSW joint.
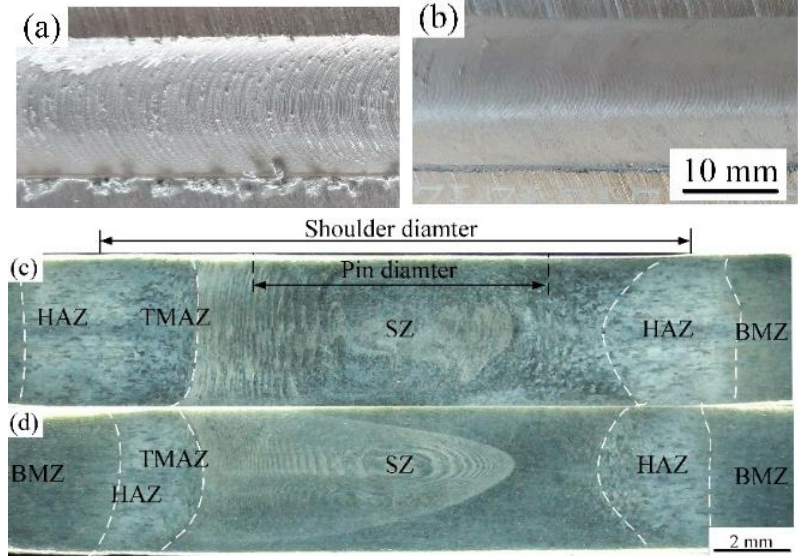

Figure 3. Macrostructural characteristics of BT-FSW and WBT-FSW joint: Surface appearances of (a) BT-FSW and (b) WBT-FSW joints, cross-sections of (a) BT-FSW and (d) WBTFSW joints

Figure 4 displays the grain morphologies in the SZs of the BT-FSW and WBT-FSW joints. For both BT-FSW and WBT-FSW, dynamic recrystallization occurs in the SZ owing to high temperature and severe plastic deformation. As a result, fine and equiaxed grains are formed in this zone. The size of the recrystallized grain can be calculated according to the following equation [20]:

$$
D^{2}-D_{0}^{2}=A \exp (-Q / R T) t_{g}
$$

where $D_{0}$ and $\mathrm{D}$ are the initial and final recrystallized grain size, $A$ is a constant, $Q$ is the activation energy for grain growth, $\mathrm{R}$ is the ideal gas constant, $T$ is the absolute temperature, and tg is the time of the grain growth. Sato et al. [20] pointed that the $D_{0}$ in the FSW process was so small that the final grain size was mainly determined by the welding temperature and the dwelling time at high temperature. For the BT-FSW, the average size in the SZ is $22.5 \mu \mathrm{m}$. By contrast, the average grain size in the SZ of the WBT-FSW decreases to $16.1 \mu \mathrm{m}$, due to the lower welding temperature and shorter dwelling time at high temperature.

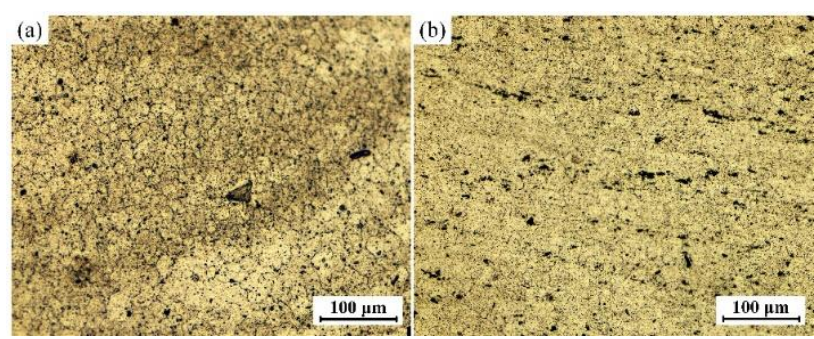

Figure 4. Grain morphologies in the SZs of the (a) BT-FSW and (b) WBT-FSW joints

\subsection{Mechanical properties}

Microhardness distributions on the cross-sections of the BT-FSW and the WBT-FSW joints are shown in Figure 5. Owing to the welding heat effect, precipitate deterioration occurs in the SZ, the TMAZ and the HAZ. As a result, a softening region is formed in both BT-FSW and WBTFSW joints. However, the refinement strengthening 
caused by the dynamic recrystallization in the SZ and the dislocations strengthening caused by the severe plastic deformation can partly compensate the hardness loss in this two zones. As a result, the HAZ has lower hardness and the lowest hardness values locate in the HAZ close to the TMAZ of the joint. In addition, owing to the symmetrical thermal and mechanical effects of the top and the bottom shoulders, the hardness distributes symmetrically with respect to the mid-thickness of the joint. The hardness values near the mid-thickness of the join are lower than those near the top and the bottom surface. This can be attributed to the superimposed heat effects by the two shoulders and the poor cooling condition near the mid-thickness of the sheet. For the BTFSW joint, the lowest hardness location is in the HAZ on the AS of the joint. On the contrary, the lowest hardness location for the WBT-FSW is in the HAZ on the RS of the joint. Comparing with the BT-FSW joint, the hardness values are increased and the softening region is significantly narrowed in the WBT-FSW joint. That is to say, the water mist cooling has significant positive effects on the mechanical strength of the joint.
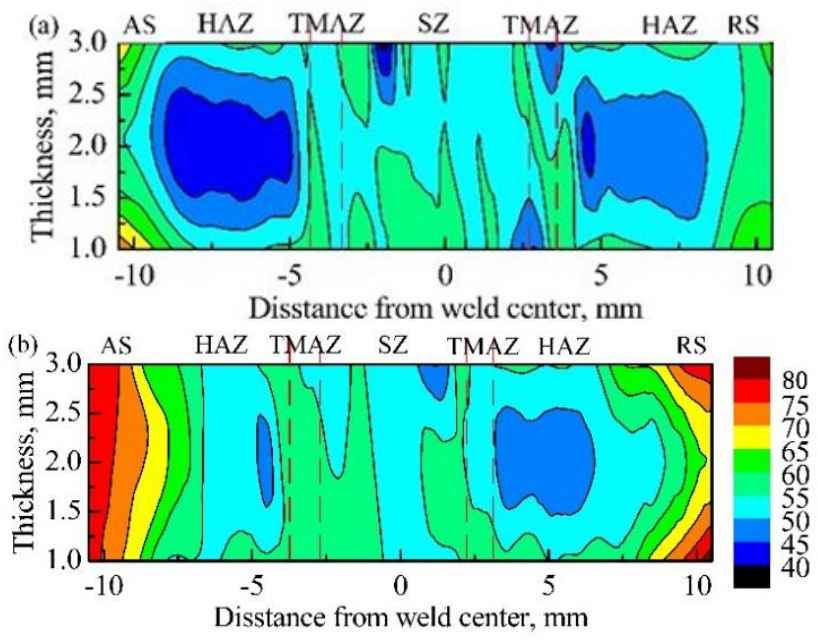

Figure 5. Microhardness distributions on the cross-sections of (a) BT-FSW and (b) WBT-FSW joints

The tensile properties of the BT-FSW and the WBTFSW joints are displayed in Figure 6. For the BT-FSW joint, the tensile strength is $170 \mathrm{Mpa}$ and the elongation is $10.1 \%$ (Figure 6a). The crack initiates and propagates in the HAZ on the AS of the joint (Figure 6b). The fracture location is in accordance with the lowest hardness location in the BT-FSW joint (Figure 5b). For the WBTFSW, owing to the water mist cooling, the tensile strength of the joint increases to $178 \mathrm{Mpa}$ and the elongation decreases to $8.7 \%$. Unlike the BT-FSW, the fracture location for the failed WBT-FSW joint is in the HAZ on the RS of the joint Figure 6c), where the lowest hardness appears (Figure 5b). Figure 6d and e show the fracture surfaces characteristics of the BT-FSW and WBT-FSW joints. The fracture surfaces of both joints are characterized by large and deep dimples, and some second phase particles can be seen at the bottom of the dimples. That is to say, both the failed BT-FSW and WBT-FSW joints experience ductile fracture.
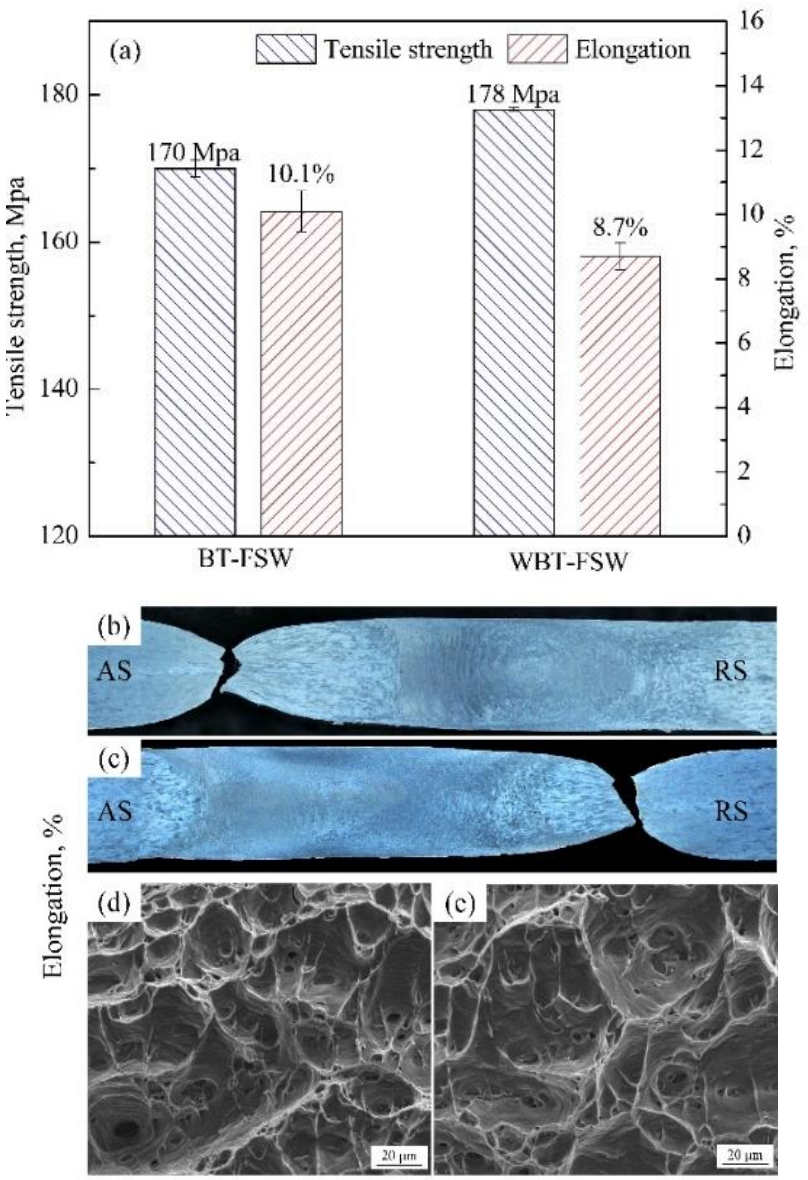

Figure 6. Tensile properties of the BT-FSW and WBT-FSW joints: (a) tensile strength and elongations of joints, fracture locations of (b) BT-FSW and (c) WBT-FSW joints, fracture surfaces of (d) BT-FSW and (e) WBT-FSW joints

\section{Summary}

In this study, a novel WBT-FSW method was developed and 6063-T6 aluminum alloy sheet was successfully jointed by WBT-FSW. Comparing with the conventional BT-FSW, the water mist cooling can significantly decrease the welding temperature in the WBT-FSW. As a result, for the WBT-FSW smoother weld surface and joint with narrower softening region can be obtained. The hardness of the WBT-FSW joint was obviously higher than that in the BT-FSW joint. The tensile strength of the WBT-FSW was $178 \mathrm{MPa}$ which was $11.4 \%$ higher than that of the BT-FSW joint.

\section{Acknowledgment}

The authors are grateful to be supported by the Implementing Innovation-Driven Development Capacity Building Special Funds project of Guangdong Academy of Sciences (2017GDASCX-0847 and 2018GDASCX0113), the Science and Technology Plan Project of Guangzhou City (201807010068 and 201704030056), and the Science and Technology Plan Project of Guangdong Province (2015B090922011). 


\section{References}

1. G. Cam, S. Mistikoglu, J. Mater. Eng. Perform. 23, 1936-1953 (2014)

2. R.S. Mishra, Z.Y. Ma, Mater. Sci. Eng. R. 50, 1-78 (2005)

3. Z. Zhang, B.L. Xiao, Z.Y. Ma, Mater. Charact. 106, 255-265 (2015)

4. W.Y. Li, T. Fu, L. Huetsch, J. Hilgert, F.F. Wang, J.F. dos Santos, N. Huber, Mater. Des. 64, 714-720 (2014)

5. H.J. Liu, J.C. Hou, H. Guo, Mater. Des. 50, 872-878 (2013)

6. L. Zhou, G.H. Li, C.L. Liu, J. Wang, Y.X. Huang, J.C. Feng, F.X. Meng, Sci. Technol. Weld. Join. 22, 438-445 (2016)

7. H. Zhang, M. Wang, X. Zhang, G. Yang, Mater. Des. 65, 559-566 (2015)

8. T.-J. Yoon, J.-G. Yun, C.-Y. Kang, Mater. Des. 90, 568-578 (2016)

9. H. Shirazi, S. Kheirandish, M.A. Safarkhanian, Measurement. 76, 62-69 (2015)

10. M.A. Wahid, Z.A. Khan, A.N. Siddiquee, Trans.
Nonferrous. Metal. Soc. Ch. 28, 193-219 (2018)

11. L. Fratini, G. Buffa, R. Shivpuri, Int. J. Adv. Manuf Technol. 43, 664-670 (2008)

12. H.J. Zhang, H.J. Liu, L. Yu, J. Mater. Eng. Perform 21, 1182-1187 (2012)

13. H.J. Zhang, H.J. Liu, L. Yu, Sci. Technol. Weld. Joining.16, 459-464 (2011)

14. H. Zhang, H. Liu, Metallography, Microstructure, and Analysis. 1, 269-281 (2012)

15. H.J. Zhang, H.J. Liu, L. Yu, Mater. Des. 32, 44024407 (2011)

16. J.Q. Su, T.W. Nelson, R. Mishra, M. Mahoney, Acta. Mater. 51, 713-729 (2003)

17. C.B. Fuller, M.W. Mahoney, M. Calabrese, L. Micona, Mater. Sci. Eng. A. 527, 2233-2240 (2010)

18. H.L. Peng Dong, Daqian Sun, Wenbiao Gong, Jie Liu, Mater. Des. 45, 524-531 (2013)

19. R.S. Mishra, Z.Y. Ma, Mater. Sci. Eng. R. 50, 1-78 (2005)

20. Y. Sato, M. Urata, H. Kokawa, Metall. Mater. Trans. A. 33, 625-635 (2002) 